\title{
Intestinal intussusception in infants : Interest of ultrasound-guided hydrostatic reduction
}

\author{
Nasser Maskaoui ${ }^{3}$, Samia Laklalech $^{1,2}$, Mounir Erraji ${ }^{1,2}$, Hicham Zerhouni ${ }^{1,2}$, Fouad Ettayebi ${ }^{1,2}$, \\ and Houda Oubejja ${ }^{1,2,3,4}$ \\ ${ }^{1}$ Pediatric Surgical Emergency Service, Rabat Children's Hospital, Morocco \\ ${ }^{2}$ Faculty of Medicine and Pharmacy, Mohammed V, University, Rabat, Morocco \\ ${ }^{3}$ Biology and Health Laboratory, Faculty of Science, Ibn Tofail University, Kenitra, Morocco \\ ${ }^{4}$ Laboratory of Biostatistics, Clinical Research and Epidemiology, Faculty of Medicine and Pharmacy of Rabat, Morocco
}

\begin{abstract}
Ultrasound-guided hydrostatic reduction of acute intussusception is a nonoperative technique, simple, performed under ultrasound guidance without risk of irradiation. Purpose: Our study aimed to evaluate the efficiency and utility of Ultrasound-guided hydrostatic reduction in the management of infant intussusception. Material and Methods: We conducted a retrospective study of cases of intussusception in infants (1-24 months) treated in the pediatric surgical emergency service of Rabat children's Hospital during the period 2016-2020. Of the 135 cases collected during the study, 109 patients received ultrasound-guided hydrostatic reduction while 26 infants inderwent primary surgery. Results: This condition was most common in the age group (3-9 months) with $78 \%$ of cases. The sex ratio M/F was 2.29. The ileocolic type was the most encountered (56\%). The complete ultrasound hydrostatic reduction was achieved for 88 infants with a success rate of $81 \%$ and an average hospitalization time of 1.4 days. 21 cases (19\%) of which 16 failed hydrostatic reduction and 05 recurrences within 24 hours, were taken up by surgery with manual reduction and bowel resection for respectively 19 and 02 cases. Conclusion: Ultrasound guided hydrostatic reduction is a safe, quick and efficient method with a high success rate, a decrease in the occurrence of recurrences and complications and should be recommended first.
\end{abstract}

Keywords: intussusception, idiopathic, hydrostatic , reduction, Ultrasound.

\section{Introduction}

Intussusception is the most common cause of intestinal obstruction in infants and young children [1]. It's the penetration of an intestinal segment into the underlying segment forming a cylinder. It's responsible for an occlusive syndrome with a venous congestion and oedema that can quickly evolve into intestinal necrosis [2]. It's a surgical emergency whose prognosis depends on the early diagnosis and reduction of invagination. In the pediatric population, ileo-colic intussusception is the most common type [3].

The cause of intussusception in children is idiopathic in $90 \%$ of cases and is presumed to be related to lymphoid hyperplasia. The remaining cases are secondary to a pathological lead point such a Meckel's diverticulum or intestinal lymphoma $[4,5]$.

The clinic presentation of intussusception is generally marked by the classic triad : paroxysmal abdominal pain, vomiting and rectal bleeding [2]. Physical exam may reveal a palpable mass in the right upper quadrant or epigastric region of the abdomen [3].

The diagnosis of intussusceptions is based on imaging tools which abdominal ultrasound is very accurate with a sensitivity of $100 \%$ and a specificity of 88 [6] (Fig1-2).

Nonoperative reduction, hydrostatic or pneumatic enema, is etablished as a treatment for idiopathic intussusception [7]. Contraindications for nonoperative management are hemodynamic instability, peritonitis and/or abdominal signs of perforation on abdominal Xray [8]. Surgery is indicated if nonoperative reduction fails, if bowel is nonviable or perforated and usually if a pathological lead point has been identified [7].

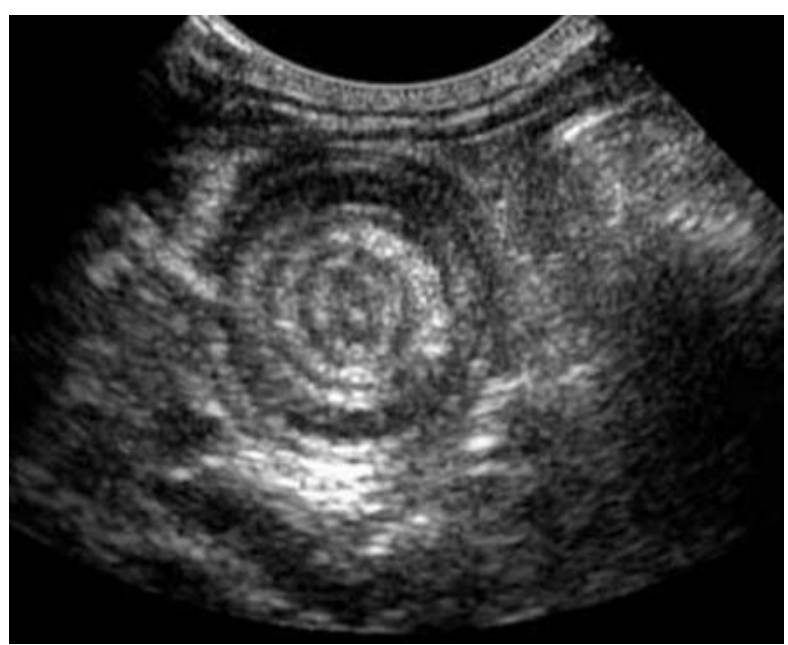

Fig 1: Ultraonographic image demonstrating the typical target shape of the intussusception 


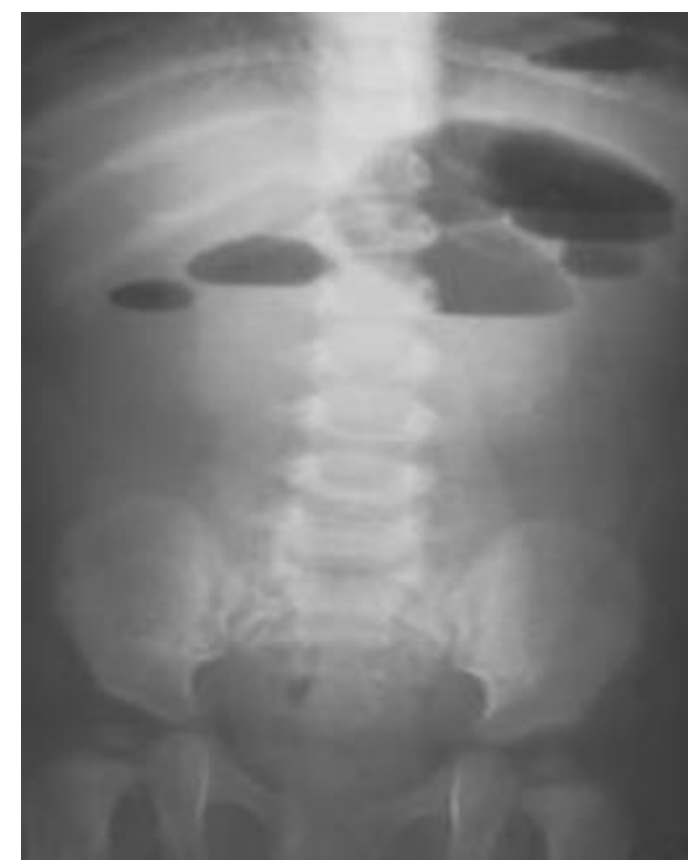

Fig 2 : Supine abdominal radiograph with multiple dilated loops typical of small bowel obstruction

Ultrasound-guided hydrostatic reduction of intussusception, was adopted for several years by the pediatric emergency service of the Children's Hospital of Rabat. It's a simple, minimally invasive method without risk of irradiation for the patient. This technique requires close collaboration between the surgeon, the radiologist and the anesthetist.

The objective of our study was to assess the effectiveness and utility of the Ultrasound-guided hydrostatic reduction in the management of infant intussusception.

\section{Technique:}

The reduction was carried out in a room equipped by the enema-can kit and the ultrasonography, with a high frequency probe, by a team composed of the pediatric surgeon, the radiologist and the anesthetist (Fig 3-4).

Infants who fulfilled the criteria for selecting the ultrasound-guided hydrostatic reduction shall first be put in condition: venous rehydratation, nasogastric tube inserted, sedation and monitoring by a multiparametric monitor. Then, a rectal catheter in appropriate size was introduced through the infant's anus; the catheter was connected by a intraveinous fluid line to an enema-can filled with isotonic saline serum pre-warmed to $37^{\circ} \mathrm{c}$ and suspended at $100-120 \mathrm{~cm}$ from the bed level.

The real time ultrasound monitoring of fluid progression allowed to confirm the success of the reduction, which matchs to the flooding of the small bowel and the disappearance of the invagination pudding. A second attempt was made 20-30 minutes in case of an incomplete reduction or failure of the first attempt. Abdominal ultrasound monitoring also allowed to detect possible recurrences during the next 24 hours. Surgical treatment was indicated in case of failure of reduction after two attempts or recurrence of intussusception.

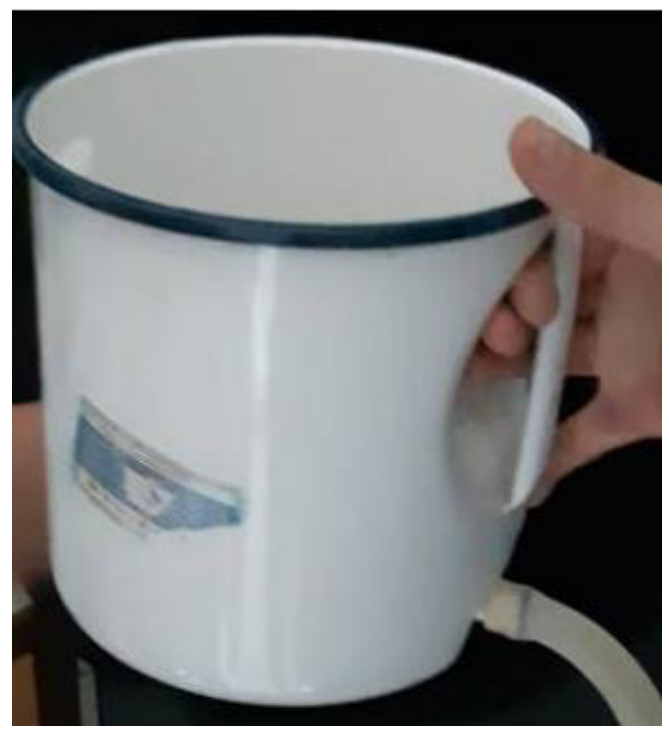

Fig 3: Enema-can used in the pediatric surgical emergency service

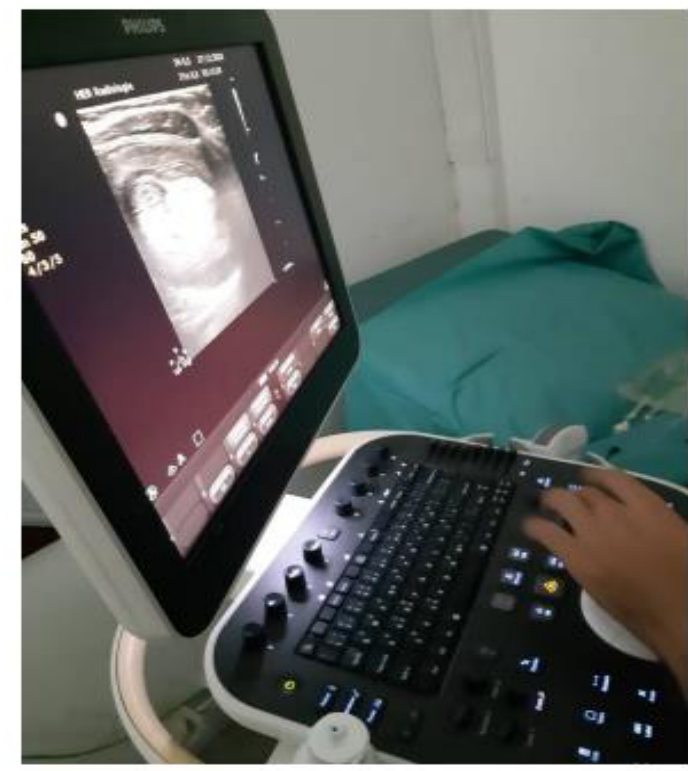

Fig 4: The ultrasonography used for guidance of the hydrostatic reduction in pediatric surgical emergency service

\section{Methods}

We conducted a retrospective and descriptive study of infant's intususception cases treated during the period 2016-2020 in the pediatric surgical emergency service of 
the Rabat Children's Hospital of Ibn Sina University Hospital Center.

Hospital registers and patient medical files were used for data collection. The inclusion criteria were:

- Idiopathic intussusception;

- Infants aged 1 to 24 months ;

- Diagnosis of intussusception confirmed by abdominal ultrasound.

Secondary intussusception cases were excluded.

The data collected covered demographic, clinical, radiological, therapeutic and prognostic characteristics.

We used the data analysis function of Microsoft excel professional plus 2016 and the categorical descriptive data were reported as numbers $(\mathrm{N})$ and percentages $(\%)$.

The published results are preliminary for a doctoral thesis topic and the ethics committee is processing the request. Nevertheless, our study was carried out in accordance with the rules of ethics and the oral informed consent was obtained from parents.

Of the 135 cases collected during the study, the ultrasound-guided hydrostatic reduction was performed for 109 infants while 26 received primary surgery.

\section{Results}

During the period of our retrospective study, 109 cases of idiopathic intussusception confirmed by abdominal ultrasound were treated with the ultrasound-guided hydrostatic reduction.

The age group most affected was between 3 and 9 months (54\%) with a mean age of 9 months and a sex ratio M/F of 2.29. The time between onset of symptoms and admission to the emergency service was less than 48 hours in $53 \%$ of cases. The most common symptoms were paroxysmal pain $(93.3 \%)$, vomiting $(71.1 \%)$ and rectal bleeding (65.9\%). This classic triad was complete in $46 \%$ of cases. Food refusal and diarrhea were found respectively in $16.3 \%$ and $11.1 \%$ of cases. $19 \%$ of cases had an alteration of the general condition. Physical exam revealed abdominal distension (16.3\%) and abdominal mass in $23.7 \%$ of cases (Table I).

\begin{tabular}{lcc}
\hline $\begin{array}{l}\text { Table I : Demographic and clinic characteristics of } \\
\text { patients presenting intussusception, Pediatric surgical } \\
\text { emergencies service, Children's Hospital of Rabat } \\
\text { (N=135) }\end{array}$ & \multicolumn{1}{c}{. } \\
\hline Age (months) & 3 & 2,2 \\
$<3$ & 73 & 54 \\
$3-9$ & 33 & 24,4 \\
$>9-12$ & 26 & 19,3 \\
$>12-24$ & & \\
Sex & 94 & 69,6 \\
Male & 41 & 30,4 \\
Female & & \\
\hline Duration of symptoms (hours) & \\
\hline
\end{tabular}

\begin{tabular}{lcc}
\hline$<24$ & 40 & 29,6 \\
$24-48$ & 32 & 23,7 \\
$>48$ & 63 & 46,7 \\
Symptoms & & \\
$\quad$ Abdominal pain & 126 & 93,3 \\
\hline Vomiting & 96 & 71,1 \\
\hline Rectal bleeding & 89 & 65,9 \\
Food refusal & 22 & 16,3 \\
\hline Diarrhea & 15 & 11,1 \\
Constipation & 9 & 6,7 \\
Clinic signs & & \\
Deterioration of general & 26 & 19,3 \\
condition & & \\
Distension & 22 & 16,3 \\
\hline Abdominal mass & 32 & 23,7 \\
Location of intussusception & & \\
$\quad$ Ileocolic & 75 & 56 \\
\hline Small bowel & 14 & 10 \\
\hline Ileoileocolic & 12 & 9 \\
\hline Unspecified & 33 & 25 \\
\hline
\end{tabular}

Abdominal ultrasound confirmed the diagnosis of intussusception in $99.3 \%$ of cases with predominance of ileocolic type (56\%), small bowel (10\%) and ileoileocolic $(9 \%)$.

The ultrasound-guided hydrostatic reduction of intussusception was complete for 88 infants, with a success rate of $81 \%$, of which 05 required a second attempt $(5.7 \%)$. Hydrostatic reduction failure was recorded for 16 infants $(14.7 \%)$ and 05 recurrences were detected within 24 hours $(4.6 \%)$. Of the 21 cases that were taken back by surgery, a manual reduction with appendectomy in principle was performed for 19 cases (14 failures and 05 recurrences) while a bowel resection was required for 02 cases following the discovery of perforation (Table II).

\begin{tabular}{|c|c|c|}
\hline \multicolumn{3}{|c|}{$\begin{array}{l}\text { Table II: Methods and results of treatment of patients } \\
\text { presenting Intussusception, Pediatric surgical emergencies } \\
\text { service, Children's Hospital Of Rabat }(\mathrm{N}=135)\end{array}$} \\
\hline Treatment & $\mathrm{N}$ & $\%$ \\
\hline \multicolumn{3}{|l|}{ Primary surgery ( 26 cases } \\
\hline Acute bowel obstruction & 25 & 96,15 \\
\hline $\begin{array}{l}\text { Alteration of general } \\
\text { condition }\end{array}$ & 1 & 3,85 \\
\hline \multicolumn{3}{|l|}{$\begin{array}{l}\text { Hydrostatic reduction } \\
\text { (109 cases) }\end{array}$} \\
\hline Success & 88 & 80,7 \\
\hline Failure & 16 & 14,7 \\
\hline $\begin{array}{l}\text { Recurrence after } 24 \\
\text { hours }\end{array}$ & 5 & 4,60 \\
\hline \multicolumn{3}{|l|}{$\begin{array}{l}\text { Surgical reduction ( } 21 \\
\text { cases) }\end{array}$} \\
\hline Manual reduction & 19 & 90,5 \\
\hline Bowel resection & 2 & 9,5 \\
\hline
\end{tabular}

The mean duration of hospital stay was 2.3 days for all patients with an interval between 1 and 10 days. 


\section{Discussion}

Intussusception is the most common abdominal emergency of early childhood [9]. The prognosis is generally favorable if the treatment is provided early. If the intussusception is not relieved, the vascular supply of the bowel may be compromised, resulting in intestinal ischemia and possibly perforation. Untreated, intussusception may be fatal [10].

In our series, data on age, sex, clinical, duration of symptoms, and location of intussusception were generally comparable to data from other studies $[11,12,13]$. The clinical presentation of intussusception is variable depending on the time taken to seek care, the way signs are collected or the perception of pain by parents [14]. The frequency of functional signs differs according to the geographical area and the classic triad (vomiting, rectal bleeding, abdominal pain) was found between 10 and $82 \%$ of cases [1]. In our series, it was present in only $46 \%$ of cases. The diagnosis of intussusception should be considered by the practitioner for any suggestive clinical sign without looking for the classic triad to avoid delays in treatment.

Nonsurgical treatment of intussusception is possible in most cases, especially at institutions with pediatric surgery facilities and personnel for use as a backup [15]. The ultrasound-guided hydrostatic reduction is used in the pediatric emergency service of the Children's Hospital of Rabat. It's considered one of the most promising nonsurgical treatment methods of pediatric intussusception with a high success rate in properly selected patients [12]. For several studies, this procedure was safe, simple, effective, economical, less time consumming with few complications, no radiation hazard and a minimal hospital stay $[11,12,13]$.

Abdominal ultrasound has become the gold standard to diagnose ou exclude intussusception. It allow to improve the precocity and the diagnosis precision, to evaluate the prognosis and to guide the treatment [16].

Contraindications to a hydrostatic reduction include an absent or a scattered vascularity within the lesion on colour Doppler studies, evident signs of perforation and peritonitis, ascites, multiples intussusception and recurent intussusceptions with known pathological points [11,13].

In our study, the complete ultrasound guided hydrostatic reduction was achieved for 88 infants, with 05 at the second attempt representing a success rate of $80.7 \%$. This rate was comparable to the rates found in the literature, which varied between 80 and $91 \%$ in published studies $[11,13,17,18]$.

The failure of hydrostatic reduction was recorded for 16 cases $(14.7 \%)$ and 05 recurrences $(5.7 \%)$ were detected in the following 24 hours. The risk factors associated significantly with the failure of hydrostatic reduction varied by studies published. Fallon et al [19] reported that the age under one year was significantly associated with the failure of the reduction; According to Reijnen et al [20], longer duration of symptoms ( $>$ or equal to 48 hours) was a predictor of a low success rate. He et al. [21] concluded that the location of invagination (left abdomen), rectal bleeding and peritoneal fluid were risk factors associated with the failure of the reduction.

In our study, $46.7 \%$ of the cases had a duration of symptoms before admission more than 48 hours and $80.7 \%$ of the cases were aged less than 1 year which might explain the cases of failure recorded. However, the risk factors for hydrostatic reduction failure have not been analyzed in our series and will be studied in the future publication.

Surgical treatment was indicated for 21 cases and bowel resection was performed for two cases following the discovery of a perforation with a rate of $1.8 \%$. This rate was less than the results of Daneman et al [22] which reported a perforation rate of $0-5.9 \%$ for both reduction methods (hydrostatic and pneumatic).

The prognosis was favorable for all cases in our series with a decrease of the mean duration of hospital stay for patients treated by hydrostatic reduction, which was 1.4 days or 33.6 hours compared to those treated by surgery (3.47 days). This duration was less than the data from the study by Xialong Xie et al [23] which reported an mean hopital stay of 69.06 hours.

\section{Conclusion}

Ultrasound guided hydrostatic reduction is a simple, quick and safe method whitout risk of irradiation for infants. Our study found that this technique had a high success rate, a decrease in the occurrence of complications and recurrences with minimal hospital stay. In the absence of contraindications, it should be recommended in the first place for idiopathic intussusception of the infant. Close collaboration between the pediatric surgeon, the radiologist and the anesthetistt is essential for early and adequate care.

\section{References}

1. J. Bines, B. Ivanoff, Acute intussusception in infants and children : incidence, clinical presentation and management : a global perspective. Report 2.19, Geneva, World Health Organisation (2002), https://apps.who.int/iris/handle/10665/67720

2. S. Franchi, H. Martelli, A. Paye-Jaouen, D. Goldszmidt, D. Pariente, Acute intestinal intussusception in infants and children, EMC-Pediat 2, 45-57 (2005). 
3. P. Marsicovetere, S.J. Ivatury, B. White, S.D. Holubar, Intestinal Intussusception : Etiology, Diagnosis, and Treatment, Clin Colon Rectal Surg 30, 30-39 (2017).

4. L. Bajaj, M.G. Roback, Postreduction Management of Intussusception in a Children's Hospital Emergency Department. Pediatrics 112(6), 1302-1307 (2003).

5. M.D. Stringer, S.M. Pablot, R.J. Brereton, Paediatric intussusception, Br.J.Surg 79, 867-876 (1992).

6. P. Verschelden, et al, Intussusception in Children : Reliability of US in Diagnosis- A prospective study, Radiology 184, 741744 (1992).

7. B. Bekdash, S.S. Marven, A. Spring, Reduction of intussusception: defining a better index of successful non operative treatment, Pediatr Radiol 43, 649-656 (2013).

8. J. Khorana, et al, Enema reduction of intussusception: The success rate of hydrostatic and pneumatic reduction, Ther Clin Risk Manag 11, 1837-1842 (2015).

9. G.S. Bisset III, D.R. Kirks, Intussusception in infants and children: Diagnosis and therapy, Radiology 168, 141-145 (1988).

10. J. Jiang, et al, Childhood intussusception: A literature review, Plos One 8(7), e68482 (2013).

11. M.M. Ahmad, et al, A experience of ultasound-guided hydrostatic reduction of intussusception at a tertiary care center, S Afr J Surg 54(1), 10-13 (2016).

12. Krishnakumar, et al, Ultrasound guided hydrostatic reduction in the management of intussusception, Indian $\mathrm{J}$ Pediatr, 73(3), 217-220 (2006).

13. S.M. Digant, S.Rucha, D, Ekta, Ultrasound guided reduction of an ileocolic intussusception by a hydrostatic method by using normal saline enema in peadriatric patients: Astudy of 30 cases, J Clin Diagn Res 6(10), 1722-1725 (2012).
14. P. Minodier, T. Merrot, Invagination intestinale aigue de l'enfant. Med. Ther. Pediatrie, 9(1), 29-34 (2006).

15. G. Del-Pozo, et al, Intussusception in children: Current concepts in diagnosis and enema reduction, Radiographics $\mathbf{1 9}$, 299-319 (1999).

16. C.Baud, et al, Diagnostic échographique d'une invagination intestinale aigue chez l'enfant et impact thérapeutique, JIDI, 2, 135-142 (2019).

17. C.WY. Wong, et al, Childhood intussusception: 17-year experience at a teriary referral centre in Hong Kong, Hong Kong Med J 21(6), 518-523 (2015).

18. X. Xialong, et al, Risk factors for failure of hydrostatic reduction of intussusception in pediatric patients: A retrospective study, Medicine 98(1), e13826 (2019).

19. S.C. Fallon, et al, Risk factors for surgey in pediatric intussusception in the era of pneumatic reduction, J Pediatr Surg 48, $1032-1036$ (2013).

20. J.A.M. Reijnen, et al, Intussusception : Factors related to treatment, Arch Dis Child 65, 871-873 (1990).

21. N. He, et al, Risk factors associated with failed sonographically guided saline hydrostatic intussusception reduction in children, J Ultrasound Med 33, 1669-1675 (2014). 22. A.Daneman, et al, Perforation during attempted intussusception reduction in children- a comparison of perforation with barium and air, Pediatr Radiol 25(2), 81-88 (1995.)

23. X. Xialong, et al, A randomized trial of pneumatic reduction versus hydrostatic reduction for intussusception in pediatric patients, J Pediatr Surg 53(8), 1464-1468 (2018). 